\title{
ÍNDICE DE INOVAÇÃO E APRENDIZAGEM E SEUS FATORES CONDICIONANTES DO ARRANJO PRODUTIVO LOCAL DE APICULTURA NO NORDESTE PARAENSE
}

\author{
Edney Saraiva Monteiro \\ Mestre em Economia Rural pela Universidade Federal do Ceará - UFC \\ edneysm@gmail.com (Brasil)
}

\begin{abstract}
Ahmad Saeed Khan
Doutor em Economia Agrícola e Recursos Naturais pela Oregon State University, Estados Unidos Professor da Universidade Federal do Ceará - UFC

saeed@ufc.br (Brasil)
\end{abstract}

\section{Eliane Pinheiro de Sousa}

Doutora em Economia Aplicada pela Universidade Federal de Viçosa - UFV

Professora do Departamento de Economia da Universidade Regional do Cariri - URCA

pinheiroeliane@ hotmail.com (Brasil)

\section{RESUMO}

Este artigo se propõe a mensurar o índice de inovação e aprendizagem das empresas apícolas que fazem parte do Arranjo Produtivo Local de apicultura do Nordeste paraense e identificar os fatores condicionantes que influenciam os níveis de inovação e aprendizagem desses apicultores. A mensuração foi feita por meio da elaboração de um índice que mede o nível de inovação e aprendizagem. Para identificar os condicionantes, utilizou-se o modelo de regressão quantílica. Os resultados mostram que a grande maioria das empresas apícolas possui baixo nível de inovação e aprendizagem, sendo que os indicadores relativos às fontes externas de informação e introdução de inovações exerceram as maiores contribuições na composição do índice e outras fontes de informação registrou o pior resultado. Ademais, conclui-se, por meio do modelo de regressões quantílicas, que o nível de escolaridade, o número de colmeias povoadas e a participação e, ou conhecimento sobre algum tipo de programa ou ações específicas para o segmento apícola promovido pelo governo federal exerceram efeitos positivos sobre o índice de inovação e aprendizagem dos apicultores em todos os quantis avaliados.

Palavras chave: Indicadores; Determinantes; Regressão quantílica; Produção de mel. 


\section{INTRODUÇÃO}

O agronegócio representa em torno de um terço do Produto Interno Bruto (PIB) brasileiro, sendo, assim, considerado um setor muito importante da economia. De acordo com o Serviço Brasileiro de Apoio às Micro e Pequenas Empresas (SEBRAE, 2006), atualmente, um dos maiores desafios desse setor é identificar e promover atividades produtivas que sejam "inclusivas" sob os aspectos tecnológicos e gerenciais, isto é, que permitam uma "difusão tecnológica", democratizando e viabilizando a incorporação das inovações nas atividades de pequenas propriedades rurais.

Dentre tais atividades, destaca-se a apicultura, que pode ser considerada alternativa importante de geração de renda para os agricultores familiares. Consiste em uma atividade de fácil manutenção, baixo custo inicial e sustentável do ponto de vista social, econômico e ambiental (Ponciano, Golynski, Souza, Ney, \& Ney, 2013).

Segundo o entendimento de Dallemole, Faria, Colman e Gomes (2010), somente recentemente, de fato, a atividade foi vista como real contribuição ao desenvolvimento rural, embora o setor apícola tenha sido implantado há bastante tempo no Brasil, onde saltou, em determinadas situações, de uma simples atividade complementar para uma atividade de caráter empresarial. Para Coronel, Sousa, e Amorim (2011), mesmo o Brasil não estando entre os maiores exportadores, percebe-se um crescimento de sua participação no cenário mundial.

Conforme dados do Instituto Brasileiro de Geografia e Estatística (IBGE, 2014), a produção nacional de mel está concentrada nos estados do Nordeste e do Sul. Entretanto, São Paulo aparece nas estatísticas por importar mel de outras regiões e exportar para outros países, isto porque processadores locais se organizaram rapidamente para aproveitar oportunidades no mercado externo a partir de 2001, desbancando o então estado líder do sul brasileiro, Santa Catarina.

Embora a região Sul detenha a maior participação percentual na produção nacional, estudos da Confederação Brasileira de Apicultura (CBA, 2013) relatam que esta foi a região com menor crescimento em termos de produção entre os anos de 1990 e 2011. Ademais, neste período, as duas principais regiões que apresentaram crescimento vertiginoso foram a região Nordeste $(848,96 \%)$ e a região Norte $(1.260,35 \%)$.

Em face do mérito do crescimento produtivo do Norte brasileiro, Both, Kato, e Oliveira (2009) relatam que a região detém um reconhecido potencial para o desenvolvimento da apicultura por meio da exploração da grande disposição natural de flora. No entanto, Silva, Venturieri, e Silva (2006) destacam que, apesar do potencial, o segmento apícola dessa região ainda não se tornou expressivo no âmbito nacional devido apresentar alguns problemas de nível organizacional (baixo nível de 
coordenação do arranjo), tecnológico (carência de equipamentos e técnicas avançadas na atividade) e mercadológico (dificuldades na colocação do mel paraense nas prateleiras dos principais mercados locais).

Dentre os estados do Norte, a Confederação Brasileira de Apicultura (CBA, 2013) aponta que o Pará tem despontado como o maior produtor de mel da região. Segundo a Federação das Associações dos Apicultores do Estado do Pará (FAPIC, 2006), a região Nordeste paraense mostra-se atrativa por apresentar atributos como: vantagens locacionais e demanda de mel superior à oferta local e regional; potencial para ocupar mão de obra e redistribuir renda; diversificação da produção; plena sustentabilidade ambiental; e existência de um Arranjo Produtivo Local (APL) especializado na produção de mel orgânico.

No entanto, como o APL de apicultura é recente, existem alguns entraves que impedem o pleno desenvolvimento da atividade no arranjo. Os principais itens definidos pela Secretaria de Agricultura do Estado do Pará (Sagri, 2007) foram: a falta de acompanhamento e assistência técnica sistemática e especializada junto aos produtores, a desorganização do sistema de produção, a deficiência estrutural (casa do mel) e a carência de inovações tecnológicas.

No tocante à carência de inovações tecnológicas, sabe-se que tais práticas são fundamentais para o crescimento das empresas em um ambiente cada vez mais concorrido e, neste quesito, segundo o diagnóstico realizado pelo SEBRAE (2006), a carência de tecnologias de manejo, a insuficiência de equipamentos apícolas avançados, a baixa inovação nos produtos finais e a falta de informações sobre o mercado impossibilitam a avanço da apicultura paraense. Ademais, conforme Barbosa e Sousa (2013), a utilização de inovações e o acúmulo do conhecimento sobre a atividade apícola, assim como seus condicionantes reveste-se de importância, uma vez que permitem orientar a formulação de estratégias para o desenvolvimento da atividade e indicar medidas de políticas públicas que fortaleçam o setor.

Contudo, o estudo dos Arranjos Produtivos Locais surge como instrumentos de fortalecimento da produção local, envolvendo toda a dinâmica para alcançar a sustentabilidade e estimular a competitividade de determinada territorialidade, o que permitiria o progresso do setor apícola na região.

Portanto, considerando a apicultura como uma atividade produtiva e promissora, com elevado potencial no Estado do Pará, surge o interesse em se estudar essa temática por meio da análise de seu Arranjo Produtivo Local. Nesse contexto, este estudo se propõe a mensurar o índice de inovação e aprendizagem das empresas apícolas que fazem parte do Arranjo Produtivo Local de apicultura do 
Nordeste paraense e identificar os fatores condicionantes que influenciam os níveis de inovação e aprendizagem desses apicultores.

\section{METODOLOGIA}

\section{1 Área de estudo e natureza dos dados}

O Arranjo Produtivo Local (APL) de apicultura é constituído por 21 municípios do Nordeste paraense, contudo, este estudo foi realizado nos municípios de Capitão Poço (microrregião do Guamá), Ourém (microrregião do Guamá) e Igarapé-Açu (microrregião de Bragantina). A escolha desses municípios se deu em virtude de serem grandes produtores de mel do Estado e terem sido o berço do arranjo apícola paraense. De acordo com dados publicados pelo Instituto Brasileiro de Geografia e Estatística (IBGE, 2014), Capitão Poço é o maior produtor de mel do Estado do Pará com 37.850 quilogramas de mel produzidos em 2012. Ourém produziu 27.785 quilogramas de mel em 2012, sendo o segundo maior produtor de mel do Estado, e Igarapé-Açu obteve uma produção de mel de 5.000 quilogramas em 2012.

Os dados empregados neste trabalho foram coletados em 2012 a partir de pesquisa direta realizada por meio de questionário aplicado com uma amostra de 79 apicultores do APL. Utilizou-se o processo de amostragem probabilística aleatória estratificada, segundo o número de colmeias povoadas, em mini, pequenos, médios e grandes produtores de mel, conforme a classificação proposta por Fachini, Oliveira, e Veiga Filho (2013). Portanto, dada a população de 142 produtores cadastrados no Projeto Apis-SEBRAE, que desenvolveu atividades destinadas ao acompanhamento da cadeia produtiva da apicultura nos arranjos paraenses, foram entrevistados 79 apicultores, sendo 18 mini, 30 pequenos, 23 médios e 8 grandes.

\subsection{Mensuração do índice inovativo e de aprendizagem}

Para mensuração do índice de inovação e de aprendizagem (IIA) do Arranjo Produtivo Local (APL) de apicultura no Nordeste paraense, tomou-se como base o método analítico utilizado nos estudos desenvolvidos por Freitas et al. (2004), Khan, Matos, Lima (2009) e Barbosa e Sousa (2013).

O índice de inovação e aprendizagem dos apicultores (IIA) foi constituído por seis indicadores: Índice de Introduções de Inovações entre 2010 e 2012 (IIIN); Índice de Inovações Realizadas em 2012 
e sua constância (IIRE); Índice de Atividades de Treinamento e Capacitação importantes para o Aprendizado (ITCA); Índice de Fontes Internas de Informação importantes para o aprendizado (IFII); Índice de Fontes Externas de Informação importantes para o aprendizado (IFEI); e Índice de Outras Fontes de Informação importantes para o aprendizado (IOFI). Atribuíram-se pesos 0 e 1 para as variáveis componentes do IIIN, sendo que se considerou peso (0) para inovações não desenvolvidas e peso (1) para as realizadas. Os pesos das variáveis que fazem parte do IIRE variaram entre 0 a 2 , sendo que o peso (0) foi empregado se não tiver desenvolvido inovações, (1) se tiver desenvolvido de forma ocasional e (2) de forma rotineira. As variáveis pertencentes aos demais indicadores variaram entre 0 a 3, em que o peso (0) foi utilizado quando as atividades de treinamento e capacitação e fontes de informação não exerceram importância, (1) para baixa importância, (2) para média importância e (3) para alta importância.

As variáveis que fizeram parte do Índice de Introduções de Inovações entre 2010 e 2012 (IIIN) foram: inovação de produtos novos para a empresa; inovação de produtos novos para o mercado nacional; processos tecnológicos novos para a empresa; criação ou melhoria substancial do modo de acondicionamento dos produtos; inovações no desenho de produtos; implementação de técnicas avançadas da gestão; implementação de significativas mudanças na estrutura organizacional; mudanças significativas nos conceitos e/ou práticas de marketing; mudanças significativas nos conceitos e/ou práticas de comercialização; e implementação de novos métodos de gerenciamento, visando a atender normas de certificação.

Quanto ao Índice de Inovações Realizadas em 2012 e sua constância (IIRE), as variáveis consideradas foram: pesquisa e desenvolvimento (P\&D) na sua empresa; aquisição externa de P\&D; aquisição de máquinas e equipamentos que geraram melhorias tecnológicas; aquisição de outras tecnologias (softwares, entre outros); projeto ou desenho industrial de produtos ou processos novos; programa de treinamento orientado à introdução de produtos e processos; programas de gestão da qualidade ou de modernização organizacional; e novas formas de comercialização e distribuição de produtos. No que diz respeito ao Índice de Atividades de Treinamento e Capacitação importantes para o Aprendizado (ITCA), as variáveis consideradas foram: treinamento na empresa; treinamento em cursos técnicos realizados no APL; treinamento em cursos técnicos realizados fora do APL; estágios em empresas fornecedoras ou clientes; estágios em empresas do grupo; contratação de técnicos / engenheiros de outras empresas do APL; contratação de técnicos / engenheiros de empresas fora do APL; e absorção de formandos dos cursos técnicos localizados no APL ou próximo.

As fontes internas consideradas na aferição do Índice de Fontes Internas de Informação importantes para o aprendizado (IFII) foram: departamento de P\&D; área de produção; áreas de vendas 
e marketing; e serviços internos de atendimento ao cliente, enquanto as fontes externas que fizeram parte do Índice de Fontes Externas de Informação importantes para o aprendizado (IFEI) foram: outras empresas dentro do grupo; empresas associadas (joint venture); fornecedores de insumos; clientes; concorrentes; e empresas de consultoria. As outras fontes constituintes do Índice de Outras Fontes de Informação importantes para o aprendizado (IOFI) foram: Universidades; Instituições de testes, ensaios e certificações; conferências, seminários, cursos e publicações; feiras, exibições e lojas; informações de rede baseadas na internet. A escolha dessas variáveis foi baseada nos estudos de Sousa et al. (2011).

A partir dos estudos de Khan et al. (2009) e Barbosa e Sousa (2013), pode-se expressar algebricamente o índice de inovação e aprendizagem (IIA) de uma dada empresa apícola j concernente à inovação tecnológica $\mathrm{n}$ como: $I_{n j}=\sum_{i=y}^{m} \frac{\alpha_{i}}{w_{n}}$, sendo $w_{n}=\max \sum_{i=y}^{m} \alpha_{i}, \operatorname{logo} 0 \leq I_{n j} \leq 1$, em que: $\mathrm{I}_{\mathrm{nj}}$ representa o índice de cada indicador $\mathrm{n}$ da empresa apícola j; i, variáveis empregadas; $\mathrm{n}$, indicador adotado; $[\mathrm{y}, \mathrm{m}]$ correspondem às variáveis dentro do segmento i referentes ao indicador $\mathrm{n}$; $\alpha_{i}$, valor atribuído à variável $\mathrm{x}_{\mathrm{i}}$ do indicador $\mathrm{n}$; e $\frac{\alpha_{i}}{w_{n}}$ refere-se ao peso de cada variável $\mathrm{x}_{\mathrm{i}}$ na composição do índice de inovação n. Ademais, consideram-se para o indicador introdução de inovações: $n=1, i=[1$, 10] e $w_{1}=10$; para o indicador inovações realizadas: $\mathrm{n}=2, \mathrm{i}=[11,18]$ e $w_{2}=16$; para o indicador de atividades de treinamento e capacitação: $\mathrm{n}=3, \mathrm{i}=[19,26]$ e $w_{3}=24$; para o indicador de fontes internas de informação: $\mathrm{n}=4, \mathrm{i}=[27,30]$ e $w_{4}=12$; para o indicador de fontes externas de informação: $\mathrm{n}=5, \mathrm{i}=[31,36]$ e $w_{5}=18$; e para o indicador de outras fontes de informação: $\mathrm{n}=6, \mathrm{i}=$ $[37,41]$ e $w_{6}=15$.

Considerando todos os indicadores que fizeram parte da composição do índice de inovação, tem-se que o índice médio de inovação e aprendizagem da empresa j ( $I I A_{j}$ ) pode ser dado por: $I I A_{j}=\frac{1}{6} \sum_{n=1}^{6} I_{n j}$. Assim, o índice de inovação e aprendizagem geral incorporando todas as empresas apícolas pode ser indicado por: IIAG $=\frac{1}{z} \sum_{j=1}^{z} I I A_{j}$.

Esses índices variam entre zero e um, sendo que se considerou que as empresas com valores desses índices iguais ou superiores a 0,80 tenham registrado alto nível de inovação e aprendizagem; as que apresentaram valores compreendidos entre 0,50 e 0,79, nível de inovação e aprendizagem intermediário; e as empresas com valores menores do que 0,50, baixo nível de inovação e 
aprendizagem. Tais intervalos de valores foram selecionados tomando como base a especificação adotada nos estudos de Khan et al. (2009) e Barbosa e Sousa (2013).

Com o intuito de verificar a existência de diferença entre as médias do índice de inovação e aprendizagem entre os diversos portes e localizações das empresas analisadas, empregou-se o teste de Scheffé, proposto por Scheffé (1959). Esse teste é recomendado para comparações múltiplas de médias de um pequeno número de grupos e com tamanhos diferentes.

\subsection{Regressão quantílica}

Para identificar os condicionantes que influenciam o nível inovativo e de aprendizagem das empresas apícolas paraenses, utilizou-se o modelo de regressão quantílica, proposto, a princípio, por Koenker e Bassett (1978). Segundo esses autores, esse método apresenta vantagens em relação ao modelo dos Mínimos Quadrados Ordinários (MQO), pois permite a caracterização da distribuição condicional de uma variável a partir de um conjunto de regressores; possibilita empregar todos os dados para estimar os coeficientes angulares dos quantis; remove a interferência dos outliers, já que não se considera somente o efeito médio do impacto de um regressor na distribuição condicional de um regressando; e obtém estimadores mais eficientes.

De acordo com Koenker e Bassett (1978), a regressão quantílica $\theta$ pode ser representada pela seguinte equação:

$$
\min _{\beta} \frac{1}{n} \sum_{i: y_{i}>x_{t}^{\prime} \beta} \theta\left|y_{i}-x_{i}^{\prime} \beta_{\theta}\right|+\sum_{: y_{i} \leq x_{t}^{\prime} \beta}(1-\theta)\left|y_{i}-x_{i}^{\prime} \beta_{\theta}\right|=\min _{\beta} \frac{1}{n} \sum_{i=1}^{n} \rho_{\theta}\left(\mu_{\theta_{i}}\right) \text {, em que: } \rho_{\theta} \quad \text { é a }
$$

função check definida por: $\rho_{\theta}\left(u_{\theta_{i}}\right)=\left\{\begin{array}{c}\theta u_{\theta_{i}}, \mathbf{u}_{\theta_{\mathrm{i}}} \geq 0 \\ (\theta-1) u_{\theta_{i}}, \mathbf{u}_{\theta_{\mathrm{i}}}<0\end{array}\right.$.

Considerando que as variáveis explicativas não influenciam igualmente os diferentes níveis de inovação e aprendizagem das empresas apícolas paraenses, foram estimadas regressões para os quantis: 0,25; 0,50; e 0,75, com o intuito de verificar os efeitos dos condicionantes desses níveis de inovação e aprendizagem ao longo da distribuição, em que o $\theta_{t h}$ refere-se ao quantil condicional do grau de inovação e aprendizagem, podendo ser expresso por:

$$
Q_{\theta}\left(y_{i} \mid x_{1}, x_{2}, x_{3}, x_{4}, x_{5}, x_{6}, x_{7}, x_{8}\right)=\beta_{0}+\sum_{j=1}^{8} \beta_{j} x_{j}, \quad \theta \in[0,1] \text { e } j=1,2, \ldots, 8, \quad \text { em } \quad \text { que }
$$

$y_{i}$ corresponde ao índice de inovação e aprendizagem das empresas apícolas paraenses; $x_{1}$, tempo de atuação (em anos) da empresa apícola (TAE); $x_{2}$, escolaridade (em anos de estudo) do principal sócio 
fundador da empresa (ESC); $x_{3}$, idade do principal sócio fundador da empresa (IDAD); $x_{4}$, número de colmeias povoadas (COLM); $x_{5}$, participação em atividades cooperativas (COOP); $x_{6}$, participação e, ou conhecimento sobre algum tipo de programa ou ações específicas para o segmento apícola promovido pelo governo federal (POLFED); $x_{7}$, ao município de Igarapé-Açu (IGARAPE); e $x_{8}$, ao município de Ourém (OUREM).

Em relação a essas variáveis, é relevante destacar que a variável COOP corresponde a uma variável dummy, em que se assume valor um, se a empresa tiver acesso, e zero, caso contrário. Quanto à variável POLFED, admite-se valor zero, se a empresa não participar nem tiver conhecimento sobre tais programas; um, se tiver conhecimento, porém não participar; e dois, se tiver conhecimento e participar desses programas ou ações promovidos pelo governo federal.

Ademais, também foram incorporadas as dummies locacionais para verificar se há alguma diferença locacional quanto aos condicionantes do nível inovativo e de aprendizagem das empresas apícolas. A variável dummy locacional considerada referência para os demais municípios paraenses analisados foi o município de Capitão Poço. Essa escolha foi baseada no critério da maior quantidade produzida de mel no estado do Pará. Assim, todos os coeficientes estimados para as dummies locacionais devem ser avaliados como diferenciais referentes aos estimados para Capitão Poço. Portanto, as variáveis IGARAPE e OUREM assumiram valor igual à unidade se a empresa apícola pertencer ao município considerado e, zero, caso contrário.

Foram utilizados os softwares SPSS 21 e STATA 12.1, respectivamente, para operacionalização dos testes estatísticos e do modelo de regressão quantílica.

\section{ANÁLISE E DISCUSSÃO DOS RESULTADOS}

\subsection{Caracterização do nível de inovação e aprendizagem}

Conforme se verifica pela Tabela 1, mais da metade das empresas apícolas paraenses analisadas se destaca com baixo índice de treinamento e capacitação e outras fontes de informação, resultando em baixo índice de inovação e aprendizagem. A realização de treinamento e capacitação de recursos humanos deu-se por meio de cursos e palestras concernentes às formas de instalações, manejos e aproveitamento dos produtos oferecidos pela apicultura, além de visitas técnicas e estágio com outros membros da cadeia apícola e associações. Entretanto, a contratação de técnicos / engenheiros de empresas fora do arranjo e a absorção de formandos dos cursos técnicos localizados no arranjo ou nas

Revista de Administração e Inovação, São Paulo, v. 12, n.3 p. 251-267, jul./set. 2015. 
proximidades foram considerados irrelevantes e apresentando baixa importância para parcela majoritária das empresas apícolas entrevistadas no Nordeste paraense. Esses resultados refletiram no fato de nenhuma empresa ter apresentado alto ITCA e, das 79 analisadas, 50 delas terem sido classificadas com baixo ITCA.

No tocante às outras fontes de informação, os dados indicam que somente duas empresas registraram elevado IOFI e que mais de $80 \%$ apresentaram IOFI abaixo de 0,50 , podendo ser atribuído à irrelevância e à baixa importância desempenhada, sobretudo, pelas instituições de testes, ensaios e certificações, assim como pelas feiras, exibições e lojas e internet como fonte de informação para o aprendizado. As fontes internas e externas obtiveram melhores desempenhos em seus respectivos índices, uma vez que nenhuma empresa considerou o departamento de pesquisa e desenvolvimento $(P \& D)$ e a área de produção como fonte interna irrelevante de informação para o aprendizado, mas, ao contrário, exercendo, por parte da maioria entrevistada, média e alta importância para o aprendizado. Essas características estão presentes nas fontes externas provenientes de outras empresas dentro do grupo e empresas associadas.

Dentre esses indicadores analisados, as maiores participações relativas de empresas com alto índice ocorreu com IIIN e IIRE. Quanto à introdução de inovações, verificou-se, com base na pesquisa de campo, que todas as empresas realizaram inovação de produtos novos para o mercado nacional, como pólen, própolis, geleia real. Ademais, das 79 empresas apícolas entrevistadas, 67 desenvolveram mudanças significativas nos conceitos e, ou práticas de marketing. Os dados sinalizaram também que mais da metade das empresas apícolas realizaram inovação de produtos novos para a empresa, implementaram técnicas avançadas de gestão e significativas mudanças na estrutura organizacional. Todas essas inovações introduzidas geraram aumento da produtividade e da qualidade do produto, assim como redução de custos.

Em relação às inovações realizadas, a pesquisa de campo indicou que mais da metade das empresas entrevistadas desenvolveram de forma rotineira pesquisa e desenvolvimento na empresa, como o estudo do tipo de flora apícola próxima ao apiário e métodos de combate a traças e formigas; adquiriram de forma rotineira novas tecnologias; e máquinas e equipamentos, que geraram melhorias tecnológicas, como centrífuga, decantador e mesa desoperculadora; participaram de forma rotineira de programas de treinamento orientado à introdução de produtos e processos; e de programas de gestão da qualidade ou de modernização organizacional, como melhoramento das instalações dos apiários (posicionamento adequado das caixas de abelha) e correções no manejo sanitário, divisão dos enxames e substituição da abelha rainha. 


\section{Tabela 1}

Distribuições absolutas e relativas da classificação das empresas apícolas paraenses por índice de inovação e aprendizagem.

\begin{tabular}{|l|c|c|c|c|c|c|}
\hline \multirow{2}{*}{\multicolumn{1}{c|}{ Índices }} & \multicolumn{2}{c|}{ Baixo } & \multicolumn{2}{c|}{ Intermediário } & \multicolumn{2}{c|}{ Alto } \\
\cline { 2 - 7 } & $\mathrm{n}^{\mathbf{0}}$ & $\%$ & $\mathrm{n}^{\mathbf{0}}$ & $\%$ & $\mathrm{n}^{\mathbf{o}}$ & $\%$ \\
\hline Introdução de inovações (IIIN) & 33 & 41,77 & 18 & 22,78 & 28 & 35,44 \\
\hline Inovações realizadas (IIRE) & 30 & 37,97 & 38 & 48,10 & 11 & 13,92 \\
\hline Treinamento e capacitação (ITCA) & 50 & 63,29 & 29 & 36,71 & 0 & 0,00 \\
\hline Fontes internas de informação (IFII) & 39 & 49,37 & 34 & 43,04 & 6 & 7,59 \\
\hline Fontes externas de informação (IFEI) & 12 & 15,19 & 64 & 81,01 & 3 & 3,80 \\
\hline Outras fontes de informação (IOFI) & 65 & 82,28 & 12 & 15,19 & 2 & 2,53 \\
\hline Inovação e aprendizagem (IIA) & 44 & 55,70 & 35 & 44,30 & 0 & 0,00 \\
\hline
\end{tabular}

Fonte: Dados da pesquisa.

Conforme mostrado, nenhum apicultor entrevistado registrou elevado nível de inovação e aprendizagem, podendo está associado, sobretudo, ao baixo desempenho atribuído aos índices de treinamento e capacitação e outras fontes de informação.

Os dados descritos na Tabela 2 revelam que todas as grandes empresas apícolas apresentaram nível de inovação e aprendizagem intermediário, enquanto parcela majoritária dos mini e pequenos apicultores pesquisados registrou baixo nível de inovação e aprendizagem. Esses resultados indicam que se devem estabelecer medidas de políticas públicas, principalmente a essas empresas de mini e pequeno porte, possibilitando a adoção e ampliação de inovações tecnológicas e formas de aprendizagem com o intuito de torná-las mais eficiente, já que, conforme Chavas et al. (2010), o acúmulo de conhecimentos e o emprego de inovações tecnológicas propiciam maior eficiência aos sistemas produtivos. 


\section{Tabela 2}

Distribuições absolutas e relativas da classificação do índice de inovação e aprendizagem (IIA) por porte das empresas apícolas paraenses e comparações múltiplas de médias.

\begin{tabular}{|c|c|c|c|c|c|c|c|c|}
\hline \multirow[t]{2}{*}{ IIA } & \multicolumn{2}{|c|}{ Mini (I) } & \multicolumn{2}{|c|}{ Pequena (II) } & \multicolumn{2}{|c|}{ Média (III) } & \multicolumn{2}{|c|}{ Grande (IV) } \\
\hline & $\mathrm{n}^{\mathrm{o}}$ & $\%$ & $\mathrm{n}^{\mathrm{o}}$ & $\%$ & $\mathrm{n}^{\circ}$ & $\%$ & $n^{\circ}$ & $\%$ \\
\hline Baixo & 12 & 66,67 & 20 & 66,67 & 12 & 52,17 & 0 & 0,00 \\
\hline Intermediário & 6 & 33,33 & 10 & 33,33 & 11 & 47,83 & 8 & 100,00 \\
\hline Alto & 0 & 0,00 & 0 & 0,00 & 0 & 0,00 & 0 & 0,00 \\
\hline Total & 18 & 100,00 & 30 & 100,00 & 23 & 100,00 & 8 & 100,00 \\
\hline Teste & \multicolumn{2}{|c|}{ Grupos } & \multicolumn{2}{|c|}{ Diferença de médias } & \multicolumn{2}{|c|}{ Erro padrão } & \multicolumn{2}{|c|}{ Sig. } \\
\hline \multirow{12}{*}{ Scheffé } & \multicolumn{2}{|c|}{ I e II } & \multicolumn{2}{|c|}{$-0,0208$} & \multicolumn{2}{|c|}{0,0208} & \multicolumn{2}{|c|}{0,80} \\
\hline & \multicolumn{2}{|c|}{ I e III } & \multicolumn{2}{|c|}{$-0,0298$} & \multicolumn{2}{|c|}{0,0220} & \multicolumn{2}{|c|}{0,61} \\
\hline & \multicolumn{2}{|c|}{ I e IV } & \multicolumn{2}{|c|}{$-0,1508$} & \multicolumn{2}{|c|}{0,0297} & \multicolumn{2}{|c|}{0,00} \\
\hline & \multicolumn{2}{|c|}{ II e I } & \multicolumn{2}{|c|}{0,0208} & \multicolumn{2}{|c|}{0,0209} & \multicolumn{2}{|c|}{0,80} \\
\hline & \multicolumn{2}{|c|}{ II e III } & \multicolumn{2}{|c|}{$-0,0091$} & \multicolumn{2}{|c|}{0,0194} & \multicolumn{2}{|c|}{0,97} \\
\hline & \multicolumn{2}{|c|}{ II e IV } & \multicolumn{2}{|c|}{$-0,1300$} & \multicolumn{2}{|c|}{0,0278} & \multicolumn{2}{|c|}{0,00} \\
\hline & \multicolumn{2}{|c|}{ III e I } & & 298 & & 220 & &, 61 \\
\hline & & II e II & & 091 & & 194 & & 97 \\
\hline & & I e IV & & 209 & & 287 & &, 00 \\
\hline & & V e I & & 508 & & 297 & &, 00 \\
\hline & IV & & 0,1 & & & & $\overline{0,0}$ & \\
\hline & IV & & 0,1 & & & & 0,0 & \\
\hline
\end{tabular}

Fonte: Dados da pesquisa.

Verifica-se também pela Tabela 2 que, conforme esperado, a grande empresa apícola registrou o maior índice médio de inovação e aprendizagem, podendo ser decorrente do fato desse grupo de apicultores serem o que mais investiu na realização de inovações, em atividades de treinamento e capacitação, assim como pela importância dada às fontes de informação para o aprendizado. Em contrapartida, os mini apicultores tiveram a menor média do IIA, visto que grande parte dessas características não fez parte da prática adotada por tais produtores. Ademais, constata-se, com base no teste de Scheffé de comparações múltiplas de médias, que há diferença significativa nos índices médios de inovação e aprendizagem entre a grande empresa apícola e as empresas de mini, pequeno e médio porte, com $95 \%$ de confiança.

No tocante à localização, observa-se que apesar de o município de Capitão Poço liderar a maior produção de mel paraense, percebe-se, por meio da Tabela 3, que parcela majoritária $(60,61 \%)$ de suas empresas apícolas obteve baixo nível de inovação e aprendizagem. Essa classificação também prevalece em mais da metade das empresas apícolas entrevistadas do município de Ourém, indicando que não há diferença significativa nos índices médios de inovação e aprendizagem das empresas apícolas localizadas nesses dois municípios, com 95\% de confiança, considerando o teste de Scheffé. 
Com base nesse teste, constata-se ainda que o IIA médio das empresas apícolas de Capitão Poço também não é significativamente diferente das sediadas em Igarapé-Açu.

\section{Tabela 3}

Distribuições absolutas e relativas da classificação do índice de inovação e aprendizagem (IIA) por localização das empresas apícolas paraenses e comparações múltiplas de médias.

\begin{tabular}{|c|c|c|c|c|c|c|}
\hline \multirow[t]{2}{*}{ IIA } & \multicolumn{2}{|c|}{ Capitão Poço (CP) } & \multicolumn{2}{|c|}{ Ourém (O) } & \multicolumn{2}{|c|}{ Igarapé-Açu (IA) } \\
\hline & $\mathrm{n}^{\mathrm{o}}$ & $\%$ & $n^{\circ}$ & $\%$ & $n^{\circ}$ & $\%$ \\
\hline Baixo & 20 & 60,61 & 16 & 55,17 & 8 & 47,06 \\
\hline Intermediário & 13 & 39,39 & 13 & 44,83 & 9 & 52,94 \\
\hline Alto & 0 & 0,00 & 0 & 0,00 & 0 & 0,00 \\
\hline Total & 33 & 100,00 & 29 & 100,00 & 17 & 100,00 \\
\hline Teste & \multicolumn{2}{|c|}{ Grupos } & \multicolumn{2}{|c|}{ Diferença de médias } & Erro padrão & Sig. \\
\hline & \multicolumn{2}{|c|}{$\mathrm{CPeO}$} & \multicolumn{2}{|c|}{$-0,0047$} & 0,0205 & 0,97 \\
\hline & \multicolumn{2}{|c|}{$\mathrm{CP}$ e IA } & \multicolumn{2}{|c|}{$-0,0245$} & 0,0240 & 0,60 \\
\hline Scheffé & \multicolumn{2}{|c|}{$\mathrm{O}$ e CP } & \multicolumn{2}{|c|}{0,0047} & 0,0205 & 0,97 \\
\hline & \multicolumn{2}{|c|}{ O e IA } & \multicolumn{2}{|c|}{$-0,0198$} & 0,0246 & 0,72 \\
\hline & \multicolumn{2}{|c|}{ IA e CP } & \multicolumn{2}{|c|}{0,0245} & 0,0240 & 0,60 \\
\hline & \multicolumn{2}{|c|}{$\mathrm{IA} \mathrm{e} \mathrm{O}$} & \multicolumn{2}{|c|}{0,0198} & 0,0246 & 0,72 \\
\hline
\end{tabular}

Fonte: Dados da pesquisa.

A Tabela 4 apresenta a contribuição absoluta e relativa dos indicadores do índice geral de inovação e aprendizagem das empresas apícolas paraenses, mostrando a contribuição de cada indicador na formação do IIAG. Dentre os indicadores analisados, constata-se que fontes externas de informação e introdução de inovações registraram as maiores contribuições para a composição do IIAG, respectivamente, 20,47\% e 19,21\%. Por outro lado, o indicador concernente a outras fontes de informação obteve o pior resultado, correspondendo a 10,91\% na composição do índice.

De posse dos dados da Tabela 4, também se pode inferir que, em termos médios, o índice de inovação e aprendizagem das empresas apícolas paraenses configura-se como baixo, porém próximo ao nível intermediário. Verifica-se ainda que os indicadores referentes à introdução de inovações, inovações realizadas e fontes externas de informação registraram nível intermediário de inovação e aprendizagem, enquanto os demais indicadores apresentaram baixo nível de inovação e aprendizagem.

\section{Tabela 4}

Participação dos indicadores na composição do índice geral de inovação e aprendizagem (IIAG)

\begin{tabular}{|l|l|l|l|}
\hline Indicador & Valor absoluto & Valor relativo & IIAj* \\
\hline Introdução de inovações & 0,0928 & 19,21 & 0,5570 \\
\hline Inovaços realizadas & 0,0857 & 17,74 & 0,5142 \\
\hline Treinamento e capacitação & 0,0704 & 14,57 & 0,4225 \\
\hline Fontes internas de informação & 0,0826 & 17,10 & 0,4958 \\
\hline Fontes externas de informação & 0,0989 & 20,47 & 0,5935 \\
\hline Outras fontes de informação & 0,0527 & 10,91 & 0,3165 \\
\hline Total & 0,4832 & 100,00 & 0,4832 \\
\hline
\end{tabular}

Nota. * Corresponde ao índice geral de inovação e aprendizagem por índices. Fonte: Dados da pesquisa. 


\subsection{Condicionantes do nível de inovação e aprendizagem}

Para verificar os condicionantes do nível de inovação e aprendizagem dos apicultores paraenses, estimaram-se os parâmetros por meio da regressão quantílica para os quantis 0,25; 0,50 e 0,75, buscando captar a influência desses condicionantes ao longo de pontos distintos da distribuição condicional dos índices de inovação e aprendizagem e não somente na média, cujos resultados se encontram na Tabela 5.

Conforme se verifica, o parâmetro da variável tempo de atuação da empresa apícola (TAE) mostrou-se significante a $10 \%$ somente na parte inferior da distribuição dos níveis de inovação e aprendizagem e apresentou sinal positivo, indicando que o nível de inovação e aprendizagem das empresas apícolas que fazem parte desse grupo cresce com o aumento do tempo de exercício nessa atividade. Esse resultado pode ser corroborado por Barbosa e Sousa (2013) ao constatarem que a inovação tecnológica dos apicultores aumenta com o acréscimo da experiência adquirida nas técnicas apícolas, captada pelo tempo de exercício nesta atividade.

A escolaridade (ESC) contribui para a melhoria dos níveis de inovação e aprendizagem das empresas apícolas em todos os quantis avaliados, sendo que os parâmetros dessa variável se mostraram significantes a $10 \%$ no quantil 25 e a $5 \%$ nos quantis 50 e 75 . Isso sinaliza que os apicultores com maior nível de instrução dispõem de maior nível de inovação tecnológica e maior conhecimento sobre as fontes de informação utilizadas para obtenção da aprendizagem da atividade apícola. Essa relação positiva entre escolaridade e inovação tecnológica é consenso na literatura econômica, sendo observada, por exemplo, nos estudos recentes desenvolvidos por Sousa et al. (2011), Barbosa e Sousa (2013) e Sobreira (2014).

A idade (IDAD) e a participação em atividades cooperativas (COOP) não se referem a fatores relevantes para explicar as diferenças observadas nos níveis de inovação e aprendizagem dos apicultores entrevistados, uma vez que os parâmetros dessas variáveis não foram significantes em nenhuma das regressões estimadas. Resultado semelhante quanto à variável cooperativismo foi encontrado por Sousa et al. (2011) ao mencionarem que essa variável não foi considerada fator determinante para explicar as diferenças entre os níveis de aprendizagem dos produtores e, por Barbosa e Sousa (2013) ao ressaltaram que tal variável não influenciou o nível de inovação tecnológico dos apicultores.

No tocante ao número de colmeias povoadas (COLM), verifica-se a existência de uma relação positiva com os níveis de inovação e aprendizagem das empresas apícolas em todos os quantis 
estimados. Esse resultado indica que uma maior quantidade de colmeias necessita de maiores investimentos com inovação tecnológica e formas de aprendizagem. De acordo com Barbosa e Sousa (2013), esse efeito positivo garante maior competitividade.

\section{Tabela 5}

Estimativa das variáveis explicativas do nível de inovação e aprendizagem das empresas apícolas por meio do modelo de regressão quantílica.

\begin{tabular}{|c|c|c|c|}
\hline \multirow{2}{*}{ Variáveis explicativas } & \multicolumn{3}{|c|}{ Quantis } \\
\cline { 2 - 4 } & 0,25 & 0,50 & 0,75 \\
\hline $\mathrm{x}_{1}(\mathrm{TAE})$ & 0,2006 & 0,1886 & 0,2825 \\
& $(0,004)$ & $(0,005)$ & $(0,001)$ \\
\hline $\mathrm{x}_{2}(\mathrm{ESC})$ & 0,0067 & 0,0056 & 0,0041 \\
& $(0,079)$ & $(0,126)$ & $0,357)$ \\
\hline $\mathrm{x}_{3}(\mathrm{IDAD})$ & 0,0074 & 0,0088 & $(0,0101$ \\
& $(0,053)$ & $(0,018)$ & $-0,0010$ \\
\hline $\mathrm{x}_{4}(\mathrm{COLM})$ & $-0,0001$ & 0,0001 & $(0,322)$ \\
\hline $\mathrm{x}_{5}(\mathrm{COOP})$ & $(0,874)$ & $(0,874)$ & 0,0002 \\
& 0,0002 & 0,0002 & $(0,006)$ \\
\hline $\mathrm{x}_{6}($ POLFED) & $(0,001)$ & $(0,001)$ & 0,0010 \\
& $-0,0207$ & 0,0054 & $(0,967)$ \\
\hline $\mathrm{x}_{7}$ (IGARAPE) & $(0,328)$ & $0,0,788)$ & 0,0385 \\
& 0,0366 & $(0,021)$ & $0,088)$ \\
\hline $\mathrm{x}_{8}($ OUREM) & $(0,058)$ & 0,0624 & $(0,015)$ \\
\hline & 0,0969 & $(0,026)$ & 0,0385 \\
& $(0,001)$ & 0,0202 & $(0,150)$ \\
\hline
\end{tabular}

Nota. Os valores entre parênteses referem-se ao $p$-value. Fonte: Dados da pesquisa.

O efeito da participação e, ou conhecimento sobre algum tipo de programa ou ações específicas para o segmento apícola promovido pelo governo federal (POLFED) sobre os níveis de inovação e aprendizagem das empresas apícolas foi positivo e significante em toda a distribuição. Isso significa que a participação dos apicultores em projetos de âmbito federal, como o Programa de Aquisição de Alimentos (PAA) e o Programa Nacional de Alimentação Escolar (PNAE) influenciam positivamente os níveis de inovação e aprendizagem, uma vez que precisam se adequar às exigências desses programas federais. Essa relação positiva é confirmada no estudo de Sobreira (2014) ao verificar que o PAA foi capaz de diferenciar os apicultores beneficiários dos não beneficiários do programa quanto ao nível tecnológico. Portanto, esse programa influenciou a adoção de práticas e técnicas recomendadas que determinem diretamente o nível tecnológico de seus beneficiários.

Quanto às dummies locacionais, constata-se que as empresas apícolas localizadas no município de Igarapé-Açu registraram maiores níveis de inovação e aprendizagem quando comparados com as que fazem parte do município de Capitão Poço, sendo contrário ao esperado, visto que o município de 
Capitão Poço se destaca com a maior quantidade produzida de mel do Estado do Pará, onde se localiza as grandes empresas apícolas paraenses. Ademais, pode-se inferir que as empresas apícolas sediadas em Ourém apresentam os mesmos níveis de inovação e aprendizagem das localizadas em Capitão Poço, visto que os parâmetros da variável OUREM não foram significantes. Esse resultado pode está associado ao fato de utilizarem similares índices de inovação e aprendizagem.

\section{CONCLUSÕES}

As empresas apícolas paraenses que fazem parte do Arranjo Produtivo Local de Apicultura configuram-se como baixo nível de inovação e aprendizagem, sendo que, dos seis indicadores considerados, três deles (treinamento e capacitação, fontes internas de informação e outras fontes de informação) classificam-se como baixo, sendo que este último indicador deteve o pior resultado. Em contrapartida, fontes externas de informação e introdução de inovações registram as maiores contribuições para a composição do índice geral de inovação e aprendizagem.

O nível de escolaridade, o número de colmeias povoadas e a participação e, ou conhecimento sobre algum tipo de programa ou ações específicas para o segmento apícola promovido pelo governo federal exerceram efeitos positivos sobre o índice de inovação e aprendizagem dos apicultores em todos os quantis avaliados, enquanto a variável tempo de atuação da empresa apícola somente influenciou o IIA na parte inferior da distribuição.

Ademais, pode-se inferir que a idade e a participação em atividades cooperativas não representam fatores relevantes para explicar as diferenças observadas nos níveis de inovação e aprendizagem dos apicultores.

\section{REFERÊNCIAS}

Barbosa, W. F., \& Sousa, E. P. (2013). Nível tecnológico e seus determinantes na apicultura cearense. Revista de Política Agrícola, 22 (3), 32-47.

Both, J. P. C. L., Kato, O. R., \& Oliveira, T. F. (2009). Perfil socioeconômico e tecnológico da apicultura no município de Capitão Poço, Estado do Pará, Brasil. Amazônia (Banco da Amazônia), $5,7-21$.

CBA. (2013). Estatísticas de Produção e Comercialização. Retirado de http://www.brasilapicola.com.br/?q=node/100. 
Coronel, D. A., Sousa, E, P., \& Amorim, A. L. (2011). Desempenho exportador do mel natural nos estados brasileiros. Pesquisa \& Debate, 22, 343-360.

Chavas, J. P., Chambers, R. G., \& Pope, R. D. (2010). Production economics and farm management: a century of contributions. American Journal of Agricultural Economics, 92, 356-375.

Dallemore, D., Faria, A. M. M., Colman, W., \& Gomes, V. M. (2010). O Arranjo Produtivo Local da Apicultura de Mato Grosso: evolução recente e necessidade de ajustes. Revista de Estudos Sociais (UFMT), 24, 177-193.

IBGE. (2014). Pesquisa Pecuária Municipal - Produtos de origem animal. Retirado de http://www.sidra.ibge.gov.br.

Fachini, C., Oliveira, M. D. M., Veiga Filho, A. A. (2013). Análise econômica da produção de mel segundo diferentes perfis em Capão Bonito, Estado de São Paulo. Informações Econômicas, 43, 2942 .

FAPIC. (2006). O panorama da apicultura paraense. Anais. Encontro Estadual de Apicultores do Estado do Pará.

Freitas, D. G. F., Khan, A. S., \& Silva, L. M. R. (2004). Nível tecnológico e rentabilidade de produção de mel de abelha (Apis mellifera) no Ceará. Revista de Economia e Sociologia Rural, 42, 171-188.

Khan, A. S., Matos, V. D., \& Lima, P. V. P. S. (2009). Desempenho da apicultura no estado do Ceará: competitividade, nível tecnológico e fatores condicionantes. Revista de Economia e Sociologia Rural, 47, 651-655.

Koenker, R., \& Basset, G. (1978). Regression quantiles. Econometrica, 46, 33-50.

Ponciano, N. J., Golynski, A., Souza, P. M., Ney, M. G., \& Ney, V. S. P. (2013). Caracterização do nível tecnológico dos apicultores do Estado do Rio de Janeiro. Revista de Economia e Sociologia Rural, 51, 499-514.

SAGRI. (2007). Plano de desenvolvimento de apicultura da região Nordeste paraense. Belém. Retirado de http://(www.desenvolvimento.gov.br/arquivos/dwnl_1214833281.

Scheffé, H. (1959). The analysis of variance. New York: Wiley.

SEBRAE. (2006). Informações de mercado sobre mel e derivados da colmeia. Retirado de http://www.homolog.portal.sebrae.com.br.

Silva, G. F., Venturieri, G. C., Silva, E. S. A. (2006). Meliponicultura como alternativa de desenvolvimento sustentável: gestão financeira em estabelecimentos familiares no município de Igarapé-Açu, PA. Anais. XVI Congresso Brasileiro de Apicultura e II Congresso Brasileiro de Meliponicultura.

Sobreira, D. B. (2014). Avaliação dos impactos do Programa de Aquisição de Alimentos (PAA) no estado do Ceará: o caso do mel. Dissertação de Mestrado, Departamento de Economia Agrícola / Universidade Federal do Ceará, Fortaleza, Ceará. 
Sousa, E. P., Justo, W. R., \& Campos, A. C. (2011). Determinantes das escolhas inovativas e de aprendizagem dos fruticultores cearenses. Revista de Economia e Agronegócio, 9, 421-440.

\title{
INNOVATION INDEX AND LEARNING FACTORS AND THEIR ARRANGEMENT CONDITIONING PRODUCTIVE BEEKEEPING PLACE IN NORTHEAST PARAENSE
}

\begin{abstract}
This paper aims to measure the level of innovation and learning beekeeping businesses that are part of the Local Productive Arrangement of beekeeping in Northeast of Pará and to identify the factors that influence the level of innovation and learning of these beekeepers. An index was developed and used to measure the level of innovation and learning. To identify the determinants, the quantile regression model was used. The results show that the vast majority of the beekeeping companies exhibit low level of innovation and learning, with the indicators related to the external source of information and introduction of innovations contributed the most to the composition of the index and the other sources of information showed the worst result. In addition, it is concluded, that the level of schooling, the number of populated hives, participation and knowledge about a program or specific actions for the beekeeping sector promoted by the federal government exerted positive effect on level of innovation and learning of beekeepers in all quantiles evaluated.
\end{abstract}

Key-words: Indicators; Determinants; Quantile regression; Honey production.

Data do recebimento do artigo: 01/09/2014

Data do aceite de publicação: 15/05/2015

Revista de Administração e Inovação, São Paulo, v. 12, n.3 p. 251-267, jul./set. 2015. 